\title{
Classification of five kinds of moss plants with the use of Fourier transform infrared spectroscopy and chemometrics
}

\author{
Tao $\mathrm{Hu}^{\mathrm{a}, \mathrm{b}, *}$, Wen-Ying $\mathrm{Jin}^{\mathrm{c}}$ and Cun-Gui Cheng ${ }^{\mathrm{d}}$ \\ ${ }^{a}$ Faculty of Life Science and Chemical Engineering, Huaiyin Institute of Technology, Huaian, China \\ ${ }^{\mathrm{b}}$ National Special Superfine Powder Engineering Center, Nanjing University of Science and \\ Technology, Nanjing, China \\ ${ }^{\mathrm{c}}$ Department of Computer Science and Engineering, Yiwu Industrial and Commercial College, Yiwu, \\ China \\ ${ }^{\mathrm{d}}$ College of Chemistry and Life Science, Zhejiang Normal University, Jinhua, China
}

\begin{abstract}
Fourier transform infrared spectroscopy (FT-IR) with Horizontal Attenuated Total Reflectance (HATR) techniques is used to obtain the FT-IR spectra of five kinds of mosses, such as Ptychomitrium dentatum (Mitt.) Jaeg., Ptychomitrium polyphylloides (C. Muell.) Par., Ptychomitrium sinense (Mitt.) Jaeg., Macromitrium syntrichophyllum Ther. Etp. Vard., and Macromitrium ferriei Card. Sz Ther. Based on the comparison of the above mosses in the FT-IR spectra, the region ranging from 4000 to $650 \mathrm{~cm}^{-1}$ was selected as the characteristic spectra for analysis. Principal component analysis (PCA) and cluster analysis are considered to identify the five moss species. Because they belong to the homogeneous plants, and have similar chemical components and close FT-IR spectroscopy, PCA and cluster analysis can only give a rough result of classification among the five moss species, Fourier self-deconvolution (FSD) and discrete wavelet transform (DWT) methods are used to enhance the differences between them. We use these methods for further study. Results show that it is an excellent method to use FT-IR spectroscopy combined with FSD and DWT to classify the different species in the same family. FT-IR spectroscopy combined with chemometrics, such as FSD and DWT, can be used as an effective tool in systematic research of bryophytes.
\end{abstract}

Keywords: Moss, horizontal attenuated total reflectance Fourier transform infrared spectroscopy, principle component analysis, cluster analysis, Fourier self-deconvolution, discrete wavelet transform, classification

\section{Introduction}

Mosses are small, soft, non-vascular plants and typically 1-10 cm tall. They commonly grow close together in clumps or mats in damp or shady locations. They have no flowers or seeds, and their wiry stems are covered with simple leaves. At certain times, mosses produce spore capsules which may appear as beak-like capsules borne aloft on thin stalks.

Mosses are one of the important kinds of higher plants and quite a few kinds of mosses are used as medicinal plants in Asia. However, due to a large number of species, small habitus and few identifying characteristics of mosses, how to identify them effectively and quickly is deserving of research.

\footnotetext{
* Corresponding author: Tao Hu, Faculty of Life Science and Chemical Engineering, Huaiyin Institute of Technology, Huaian 223003, China. Tel.: +86 5178359 1044; E-mail: hutao126@126.com.
} 
At present, testing department of traditional Chinese medicines uses methods to identify traditional Chinese medicines, such as morphological and histological identification, source identification, microscopic identification and physical and chemical identification. Many modern analytical approaches are also applied to the identification of Chinese herbal medicines, for instance, thin layer chromatography, gas chromatography, high performance liquid chromatography and UV spectra and infrared spectrum, etc. [13].

Fourier transform infrared spectroscopy (FT-IR) is an original spectroscopic technique for investigating the functional groups of the chemical components [1,11], and it has been widely used for the identification, quality control and manufacturing process supervision of traditional Chinese medicines in recent years $[3,5,9,10,14]$. However, single FT-IR spectra analysis can not be quickly and accurately applied in the analysis and identification of complex systems like traditional Chinese medicines. Chemometric methods combined with FT-IR spectroscopy can make up the defects in single FT-IR spectra analysis $[2,4,6-8,15]$. So we choose FT-IR spectra with several chemometric methods to discriminate different plants of the same family.

Wavelet transform (WT) is an efficient tool for signal analysis, such as de-noising, data compression and pattern recognition. In the aspect of pattern recognition, it is well known as a good tool for feature extraction. WT is defined as the translation and dilation of a function, so it can enhance the location features of a signal and has multi-resolution capacity with different dilations and shifts. In multi-resolution analysis, the wavelet function under different scales results in different frequencies. Therefore, the wavelet coefficient can be viewed as the characteristics of the signals. A few features can reflect the major information of the spectra after WT and it makes the judgments intuitive and concise.

In this paper, the classification among five kinds of moss species, namely Ptychomitrium dentatum (Mitt.) Jaeg., Ptychomitrium polyphylloides (C. Muell.) Par., Ptychomitrium sinense (Mitt.) Jaeg., Macromitrium syntrichophyllum Ther. Etp. Vard. and Macromitrium ferriei Card. Sz Ther., was emphatically studied. We used FT-IR spectrometer with horizontal attenuated total reflectance (HATR) technique to obtain the FT-IR spectra. Principal component analysis (PCA) and cluster analysis were then applied to the spectra data set obtained in order to identify clusters of mosses, and discrete wavelet transform (DWT) and Fourier self-deconvolution (FSD) were used to extract the feature vectors and enhance the differences among the plants for classification.

\section{Discrete wavelet transform}

In numerical analysis and functional analysis, DWT is a wavelet transform that the wavelets are discretely sampled. As with other wavelet transforms, a key advantage it has over Fourier transforms is temporal resolution: it captures both frequency and location information. Based on this advantage, DWT has a huge number of applications in science, engineering, mathematics and computer science. Most notably, it is used for signal coding to represent a discrete signal in a more redundant form, often as a preconditioning for data compression. DWT is originated from the discretization of continuous wavelet transform (CWT) and the common discretization is dyadic. The CWT of a function or signal, for example, can be defined as

$$
W_{f}(a, b)=\frac{1}{\sqrt{a}} \int_{-\infty}^{\infty} f(t) \Psi^{*}\left(\frac{t-b}{a}\right) \mathrm{d} t
$$


where $\Psi^{*}(t)$ denotes the mother wavelet function. The parameters $a$ named as scale parameter and $b$ named as translation parameter are respectively used to control the dilation and position of the mother function.

After the dyadic discretization, the function of DWT is accordingly expressed as

$$
W_{\mathrm{dwt}}(j, k)=\frac{1}{\sqrt{2^{j}}} \int_{-\infty}^{\infty} f(t) \Psi^{*}\left(\frac{t-2^{j} k}{2^{j}}\right) \mathrm{d} t
$$

where $a$ and $b$ are replaced by $2^{j}$ and $2^{j} k$. An efficient way to implement this scheme using filters was developed in 1989 by Mallat. The original signal $f(t)$ passes through two complementary filters and emerges as low frequency and high frequency signals. The decomposition process can be iterated, with successive approximations being decomposed in tum, so that a signal can be broken down into many lower-resolution components [12].

\section{Experimental section}

\subsection{Materials and preparation}

The five kinds of moss plants were Ptychomitrium dentatum (Mitt.) Jaeg., Ptychomitrium polyphylloides (C. Muell.) Par., Ptychomitrium sinense (Mitt.) Jaeg., Macromitrium syntrichophyllum Ther. Etp. Vard. and Macromitrium ferriei Card. Sz Ther (Table 1). The plant materials were collected from Beishan and campus of Zhejiang Normal University in Jinhua, Zhejiang Province, China $\left(29^{\circ} 13^{\prime} \mathrm{N}, 119^{\circ} 35^{\prime} \mathrm{E}\right)$ and Leshan in Leshan, Sichuan Province, China $\left(29^{\circ} 40^{\prime} \mathrm{N}, 103^{\circ} 27^{\prime} \mathrm{E}\right)$ in May 2008. All sample plants were similar in terms of environmental conditions and population sizes. To remove the spectral interference from water absorption bands, all samples were dried at $55^{\circ} \mathrm{C}$ for $72 \mathrm{~h}$. All samples were ground into fine powder in an agate mortar respectively, and sieved by 200 meshes $(75 \mu \mathrm{m})$.

\subsection{Spectral measurements}

The FT-IR spectra were collected at a resolution of $2 \mathrm{~cm}^{-1}$ with 32 scans using a Thermo Electron (Madison, WI, USA) Nexus 6700 FT-IR spectrometer with a room temperature deuterated triglycine sulfate (DTGS) detector, and with a single-bounce HATR (Ge) accessory. $8.0 \mathrm{mg}$ of powdered samples was respectively placed directly about $3.14 \mathrm{~mm}^{2}$ on the center of the Ge crystal of the HATR accessory for measurement. To ensure good contact with the Ge crystal surface, all powder samples were pressed against it using a pressure tower to give the same mechanical pressure on all the samples. All the obtained spectra were auto-baseline corrected. No other sample preparation was required. Each species of all samples were measured and the averaged spectrum was used for further analysis.

Table 1

Moss plant samples

\begin{tabular}{|c|c|c|}
\hline Families & Genera & Species \\
\hline Ptychomitriaceae & Ptychomitrium Fueror. & $\begin{array}{l}\text { Ptychomitrium sinense (Mitt.) Jaeg. } \\
\text { Ptychomitrium dentatum (Mitt.) Jaeg. } \\
\text { Ptychomitrium polyphylloides (C. Muell.) Par. }\end{array}$ \\
\hline Orthotrichaceae & Macromibiuma & $\begin{array}{l}\text { Macromibiuma ferriei Card. Sz Ther. } \\
\text { Macromitrium syntrichophyllum Ther. Etp. Vard. }\end{array}$ \\
\hline
\end{tabular}




\subsection{Data analysis}

The FT-IR spectra of samples were obtained by measurement. The absorption values from different wave bands were based on the characters of the absorption value. The absorption values from different wave bands based on the characters of the absorption value were obtained by copying data method. Matlab 7.0 software was used for wavelet transformation. Daubechies wavelet, which possesses better exploration ability for signal singularity, is acted as analysis wavelet. Fourier self-deconvolution and one-dimension discrete wavelet transform were taken to different samples.

\section{Results and discussion}

\subsection{FT-IR analysis}

The feasibility of obtaining for the chemical information from plants was investigated via FT-IR spectrometry. Typical IR spectra obtained from five kinds of plants Ptychomitrium sinense (Mitt.) Jaeg., Ptychomitrium dentatum (Mitt.) Jaeg., Ptychomitrium polyphylloides (C. Muell.) Par., Macromibiuma ferriei Card. Sz Ther. and Macromitrium syntrichophyllum Ther. Etp. Vard. were shown in Fig. 1.

Because FT-IR spectra can offer useful information which represents many different functional groups of biomacromolecule, the comprehensive information of fibrin, hemicellulose, protein, carbohydrate, nucleic acid and lipid in the gametocyte is obtained from the spectra. From the Fig. 1, absorption bands in the range of 4000-900 $\mathrm{cm}^{-1}$ are due to functional groups, and the band assignments are labeled in Fig. 1 . Absorption bands located around $3400 \mathrm{~cm}^{-1}$ correspond to $\mathrm{O}-\mathrm{H}$ and $\mathrm{N}-\mathrm{H}$ stretching vibrations that mainly occur from proteins and carbohydrates. The bands around $3000 \mathrm{~cm}^{-1}$ represent $\mathrm{C}-\mathrm{H}$ stretching vibrations that are mainly caused by lipids and carbohydrates. Absorption raised from $\mathrm{C}-\mathrm{H}$ bending modes is located around $1200-1500 \mathrm{~cm}^{-1}$ but overlaps with other absorption bands within this region. Three protein absorption bands located around $1680(\mathrm{C}=\mathrm{O}), 1550(\mathrm{~N}-\mathrm{H})$ and $1250(\mathrm{C}-\mathrm{N}) \mathrm{cm}^{-1}$ are assigned as amide I, II and III bands, respectively. Absorption bands around $1745 \mathrm{~cm}^{-1}$ correspond to an isolated carbonyl group (COOR), indicating ester-containing compounds are commonly found in membrane lipid and cell wall pectin. Absorption bands in the area between 1200 and $650 \mathrm{~cm}^{-1}$, referred as the fingerprint region, are due to intra-molecular phenomena. The fingerprint region is specific for each species. Chemometrics is applied to the above absorption bands. The observed absorption bands concern with low molecular weight carbohydrates, specifically to polyols and monosaccharides.

Although some complex quantitative and qualitative differences between the species analyzed are observed, specific differences are essentially unrecognizable by the naked eyes and readily illustrated the need to employ multivariate statistical techniques for the analysis of FT-IR data. We chose principal component analysis (PCA) and cluster analysis (CLA) as the research methods to analyze the absorption bands. Because the main absorption peaks of chemical compositions in bryophytes range from 4000 to $650 \mathrm{~cm}^{-1}$, this area is the main object to analysis in this paper.

\subsection{Chemotaxonomic distinction with cluster analysis}

From Fig. 2, we can obtain a closer inspection of quantitative difference, but such complicated spectra readily illustrate the need to use multivariate statistical techniques in the analysis of FT-IR data. Therefore, the first multivariate analysis we chose is cluster analysis, which is used to investigate a perspicuous 


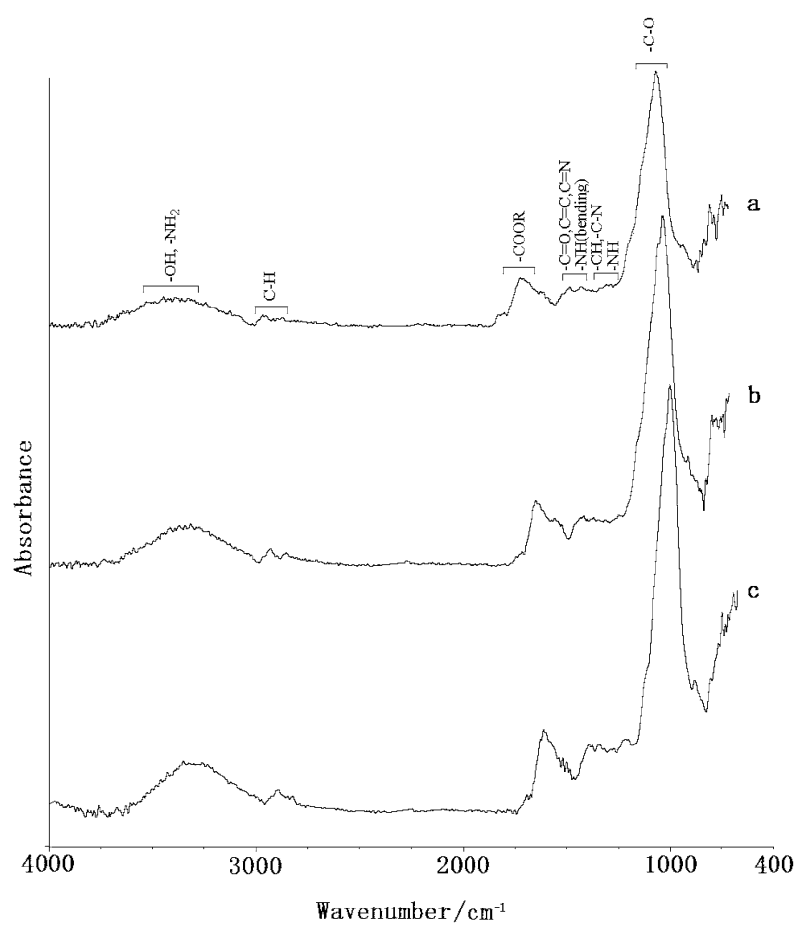

(A)

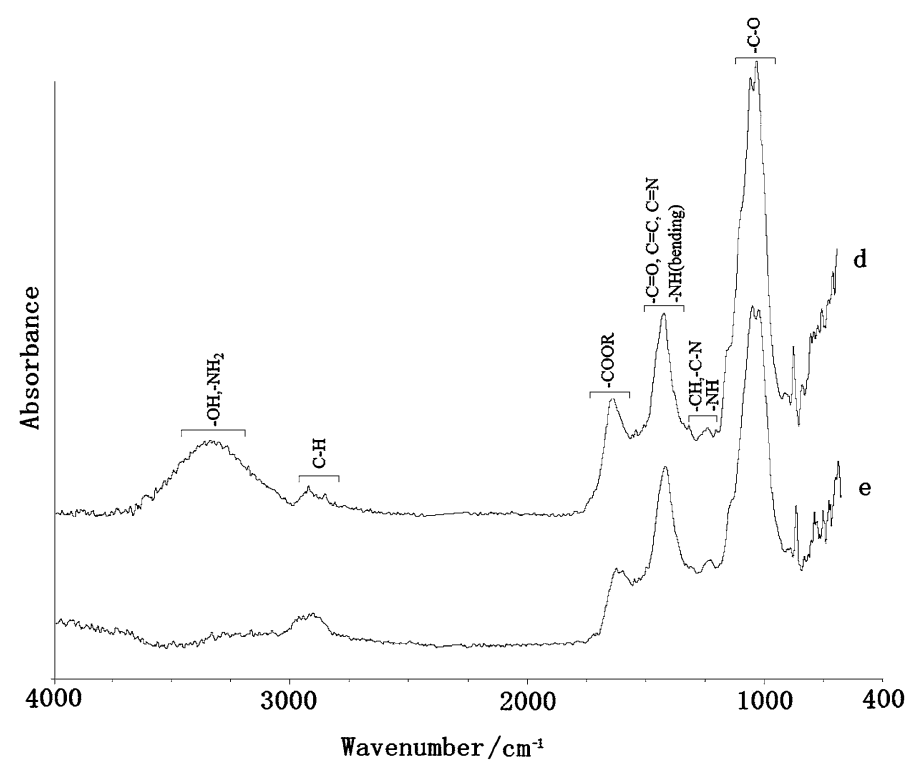

(B)

Fig. 1. (A) HATR-FT-IR spectra obtained in the $4000-650 \mathrm{~cm}^{-1}$ region for the species of Sect. Ptychomitrium Fueror. Characteristic group frequencies are indicated at the top. (B) HATR-FT-IR spectra obtained in the $4000-650 \mathrm{~cm}^{-1}$ region for the species of Sect. Macromibiuma. Characteristic group frequencies are indicated at the top. (a) Ptychomitrium sinense (Mitt.) Jaeg.; (b) Ptychomitrium dentatum (Mitt.) Jaeg.; (c) Ptychomitrium polyphylloides (C. Muell.) Par.; (d) Macromibiuma ferriei Card. Sz Ther.; (e) Macromitrium syntrichophyllum Ther. Etp. Vard. 


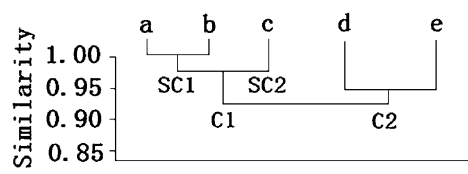

Fig. 2. Dendrogram represents the relationships between the 5 kinds of moss species clustered by hierarchical cluster analysis on the basis of FT-IR data. (a) Ptychomitrium sinense (Mitt.) Jaeg., (b) Ptychomitrium dentatum (Mitt.) Jaeg., (c) Ptychomitrium polyphylloides (C. Muell.) Par., (d) Macromibiuma ferriei Card. Sz Ther., (e) Macromitrium.

way of viewing the relationships between these taxa. Cluster analysis is a technique that clusters IR spectra on the basis of similarity with other spectra. In this study, the algorithm of paired group was used without any prior parametrization of the spectral data in the IR region $\left(4000-650 \mathrm{~cm}^{-1}\right)$. Five samples of mosses are selected to make cluster analysis. We select 24 absorption peaks in the range of the $4000-650 \mathrm{~cm}^{-1}$, and then the absorption peaks which we selected are tested by cluster analysis. The dendrogram is shown in Fig. 2.

The dendrogram divides 5 kinds of samples into two main clusters: cluster $1(\mathrm{C} 1)$ is a rather loose cluster which includes all the species in Sect. Ptychomitrium Fueror; cluster 2 (C2) comprises all the remaining species in Sect. Macromibiuma. On closer inspection, $\mathrm{C} 1$ can be seen to contain two subclusters: sub-cluster 1 (SC1) comprises Ptychomitrium sinense (Mitt.) Jaeg. and Ptychomitrium dentatum (Mitt.) Jaeg.; sub-cluster 2 is Ptychomitrium polyphylloides (C. Muell.) Par.

The results reflect clearly the real internal relationship of the five samples and give a better description of different moss species, thus it is agreed with our expectations.

\subsection{Chemotaxonomic distinction with principal component analysis}

The second multivariate analysis we used is principal component analysis (PCA), which is performed as a mathematical procedure to transform a series of possibly correlated variables into a smaller number of uncorrelated variables called principal components. The first principal component accounts for as much of the variability in the data as possible, and each succeeding component account for as much the remaining variability as possible. PCA is one of chemometric methods utilized for FT-IR analysis. Chemometric analysis of FT-IR data presents the best results for principal component analysis with the first derivative applied on samples.

The data in FT-IR spectra is analyzed by PCA. Table 2 shows the variance accounted by the first seven principal components (PCs) computed from the absorbance value of characteristic peaks used in cluster analysis. More than $95 \%$ of the total variance is explained by the first three PCs, while the other PCs are related to the insignificant part of the total variance. The PC1-PC2 scores plot ( $\sim 95 \%$ of the total variance) is reported in Fig. 3. It shows five points in Fig. 3 are dispersedly distributed, and it is hard to judge the relationship between the five moss species.

The variance distribution over the first three PCs for FT-IR spectra of five mosses is shown in Table 3. These first three PCs account for more than $98 \%$ of the total variance, and can be considered as the only important PCs to describe systematic variations. In this case, the PC1-PC2 scores plot (Fig. 3) is not sufficient to clarify the structure of the data set. A three-dimensional scores plot (Fig. 4) based on the first three PCs would be more informative and showed that the species of Macromibiuma Genus and Ptychomitrium Fueror. Genus could be grouped in separate ellipses. The result analyzed by PCA is consistent with the result of cluster analysis. 
Table 2

Variance distribution over the first four PCs calculated from the five mosses

\begin{tabular}{ccccc}
\hline & PC1 & PC2 & PC3 & PC4 \\
\hline Variance (\%) & 76.935 & 19.121 & 2.6212 & 1.3227 \\
\hline
\end{tabular}

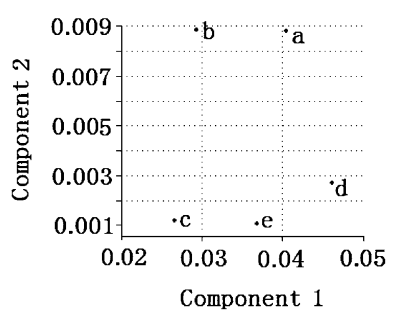

Fig. 3. Two-dimensional plots of FT-IR spectra of the 5 moss species based on PCA. (a) Ptychomitrium sinense (Mitt.) Jaeg., (b) Ptychomitrium dentatum (Mitt.) Jaeg., (c) Ptychomitrium polyphylloides (C. Muell.) Par., (d) Macromibiuma ferriei Card. Sz Ther., (e) Macromitrium syntrichophyllum Ther. Etp. Vard.

Table 3

Variance and cumulative variance for the first three PCs from PCA on the five mosses data subset

\begin{tabular}{lcc}
\hline PC & Variance $(\%)$ & Cumulative variance $(\%)$ \\
\hline PC1 & 76.935 & 76.935 \\
PC2 & 19.121 & 96.056 \\
PC3 & 2.6212 & 98.6772 \\
\hline
\end{tabular}

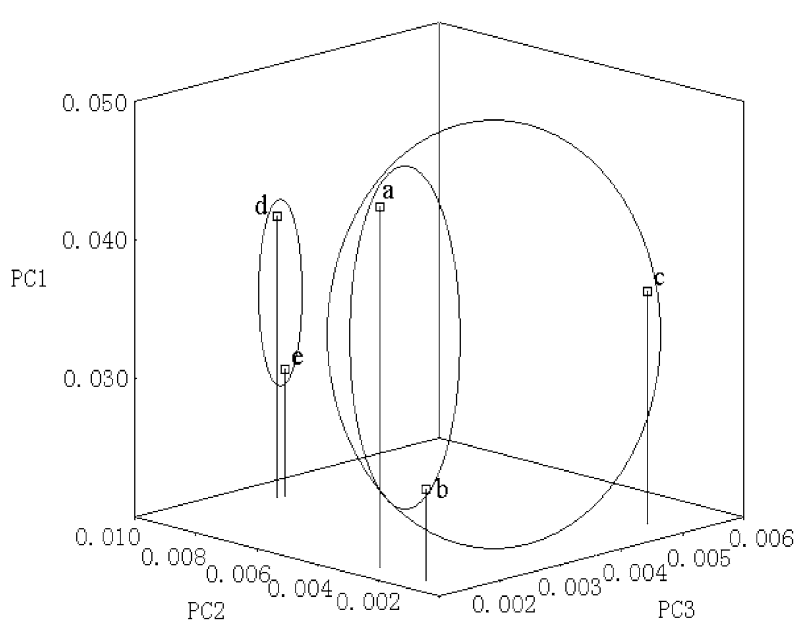

Fig. 4. Three-dimensional plot of FT-IR spectra of the 5 kinds of species based on PCA. (a) Ptychomitrium sinense (Mitt.) Jaeg., (b) Ptychomitrium dentatum (Mitt.) Jaeg., (c) Ptychomitrium polyphylloides (C. Muell.) Par., (d) Macromibiuma ferriei Card. Sz Ther., (e) Macromitrium syntrichophyllum Ther. Etp. Vard.

\subsection{Wavelet analysis of FT-IR spectra data}

As it is mentioned above, CLA and PCA are good multivariate statistical techniques to identify different genus and families of mosses. From FT-IR spectra of five mosses (Fig. 2), two species of Macromibiuma genus belong to the same genus, and they contain similar chemical composition like protein, car- 
bohydrate and plant hormones $\beta$-sitosterol, etc., whose IR absorption is quite similar, including three species of Ptychomitrium Fueror. Genus also scarcely differ from each other in their appearance. The FT-IR spectra from the different plants have very close absorbance values at the same wave number. The spectra similarity of the same genus makes it difficult to distinguish between them, so FSD and DWT were used to extract their features for further classification in our study. Generally, the region of $3600-2800$ and $1800-650 \mathrm{~cm}^{-1}$ can provide higher information of spectra. The former region includes the $\mathrm{O}-\mathrm{H}$ and $\mathrm{N}-\mathrm{H}$ stretching bands and the characterization is not obvious, while the latter region called the fingerprint region contains more molecular structural information. Therefore, we scaled the FT-IR spectra data to the same range $\left(1800-650 \mathrm{~cm}^{-1}\right)$ for FSD and DWT analysis.

\subsection{The classification of Sect. Ptychomitrium Fueror. and Sect. Macromibiuma}

According to the plant taxonomy of mosses, Sect. Ptychomitrium Fueror. and Sect. Macromibiuma belong to different families. In order to further identify them, FSD is used to deal with the FT-IR spectra. In this section, Ptychomitrium sinense (Mitt.) Jaeg. and Macromibiuma ferriei Card. Sz Ther. are selected to represent each genera, and their FT-IR spectra and FT-IR-FSD spectra are shown in Fig. 5. The 2000$1650 \mathrm{~cm}^{-1}$ region includes the fingerprint region, which contains more molecule structural information. Thus the $2000-650 \mathrm{~cm}^{-1}$ region are used to extract the spectral features.

As shown in Fig. 5(A), several absorption regions are identified, and the band assignments are labeled. The functional groups represented by the absorptions are illustrated in the above chapter. In the original FT-IR spectra (Fig. 5(A)), there are a few differences between the two species. In order to enhance the differences between Sect. Ptychomitrium Fueror. and Sect. Macromibiuma, FSD is used to deal with the FT-IR spectra. The result is shown in Fig. 5(B). From Fig. 5(B), the waveforms of the two species have been changed a lot. In the same resolution degree, Ptychomitrium sinense (Mitt.) Jaeg. (a) has some difference from Macromibiuma ferriei Card. Sz Ther. (b), the wave peaks of ester, amide I and II in Ptychomitrium sinense (Mitt.) Jaeg. and Macromibiuma ferriei Card. Sz Ther., which are quite correspondent. The amide III bands at $1250 \mathrm{~cm}^{-1}$ were found in Ptychomitrium sinense (Mitt.) Jaeg., but not in Macromibiuma ferriei Card. Sz Ther. and the shape of absorption bands and the intensity of absorption peaks in the fingerprint region, which are different. Therefore, Fourier self-deconvolution can be applied to identify different genera simply, rapidly and accurately.

\subsection{The classification of species in Sect. Macromibiuma}

The FSD was used to enhance the differences between different plants of different genera, and obtained satisfying results. So we applied this method to the study of species in the same genera. Figure 6 shows the results.

Regrettably, the results did not come up to our expectations. In the Fig. 6, as they belong to the same genus, and they contain similar chemical composition like protein, carbohydrate and plant hormones $\beta$-sitosterol, etc., their IR absorption is quite similar. The FT-IR spectra from different plants have very close absorbance values at the same wave number. The spectra similarity of the same genus makes it difficult to distinguish among them even the FT-IR spectra were transformed by FSD, so discrete wavelet transform (DWT) was used to extract their features for further classification.

Figure 7 displayed the DWT coefficients of complete stool of Macromibiuma ferriei Card. Sz Ther. and Macromitrium syntrichophyllum Ther. Etp. Vard. We make 5-level wavelet decomposition to deal with the FT-IR spectra data. Scale 1-5 in Fig. 7 indicates detailed information after decomposition. In Fig. 7, 


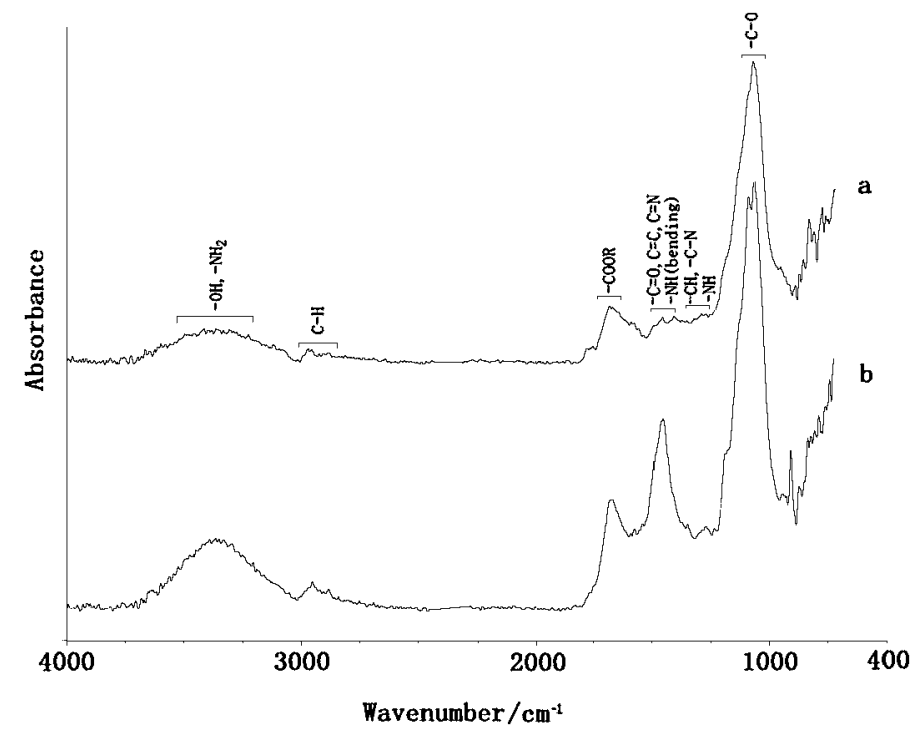

(A)

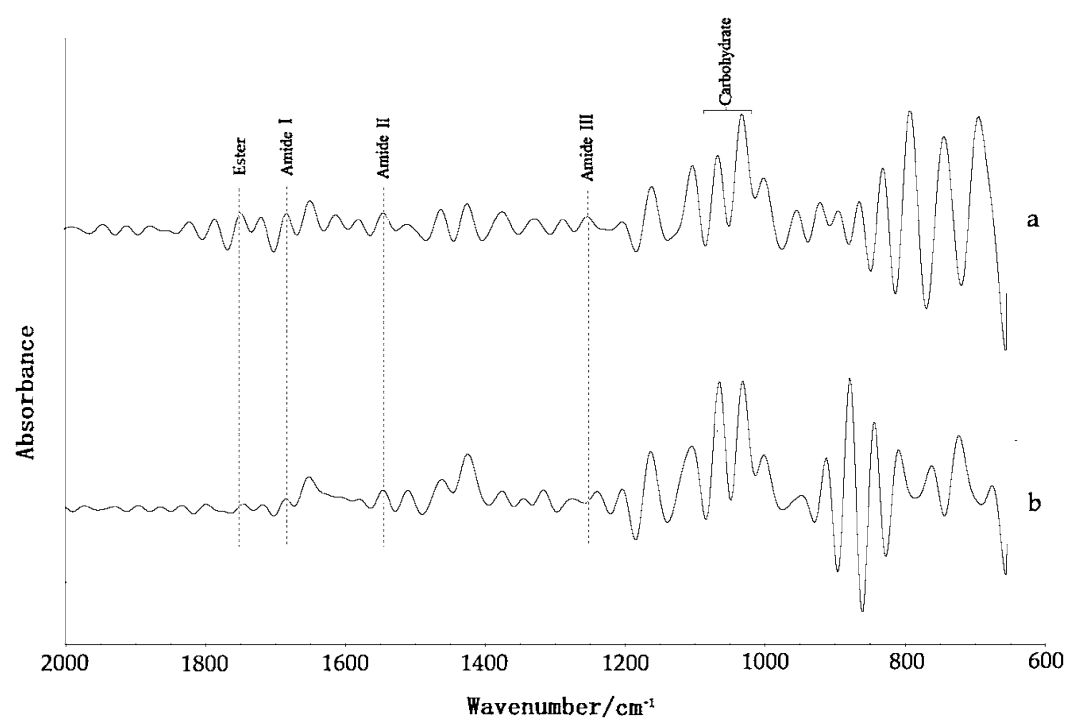

(B)

Fig. 5. (A) Absorption FT-IR spectra in the $4000-650 \mathrm{~cm}^{-1}$ region in the whole plants of Ptychomitrium sinense (Mitt.) Jaeg. (a) and Macromibiuma ferriei Card. Sz Ther. (b). Characteristic group frequencies are indicated at the top. (B) Fourier selfdeconvolution FT-IR spectra in the $2000-650 \mathrm{~cm}^{-1}$ region in the whole plants of Ptychomitrium sinense (Mitt.) Jaeg. (a) and Macromibiuma ferriei Card. Sz Ther. (b). Characteristic group frequencies are indicated at the top.

the DWT coefficients can effectively affect the information features of spectra. With the comparison of the five scales, scales 3-5 are sensitive to the change of absorption frequency in the spectra. As the DWT coefficients of Macromibiuma ferriei Card. Sz Ther. and Macromitrium syntrichophyllum Ther. Etp. Vard. have great difference in scales 3-5, different species of the same genera can be identified simply, rapidly and accurately. 


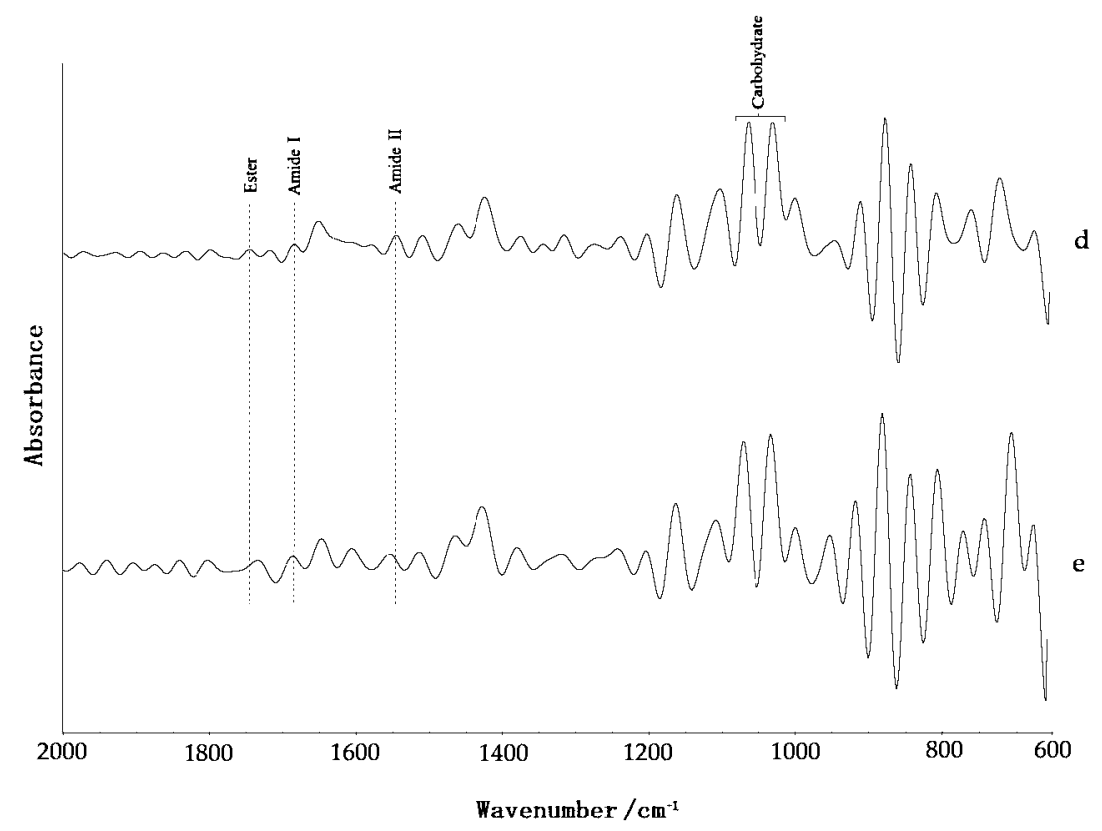

Fig. 6. Fourier self-deconvolution FT-IR spectra in the $2000-650 \mathrm{~cm}^{-1}$ region in the whole plants of two species in Sect. Macromibiuma. Characteristic group frequencies are indicated at the top. (d) Macromibiuma ferriei Card. Sz Ther. (e) Macromitrium syntrichophyllum Ther. Etp. Vard.
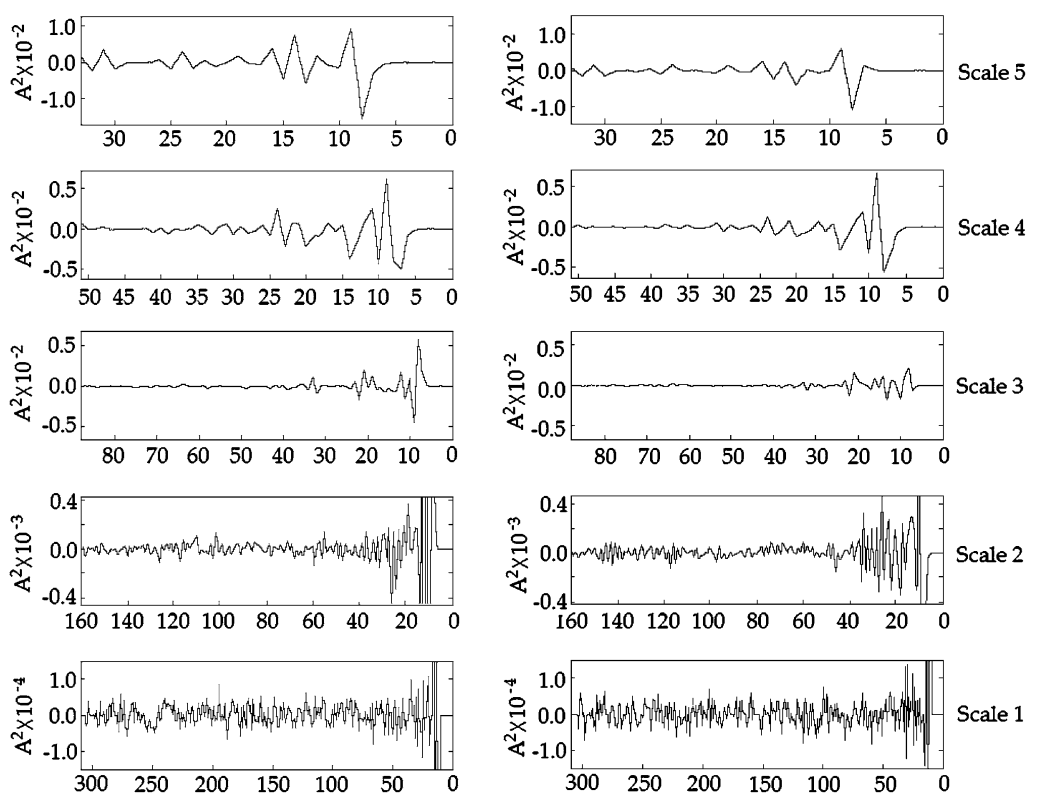

(a)

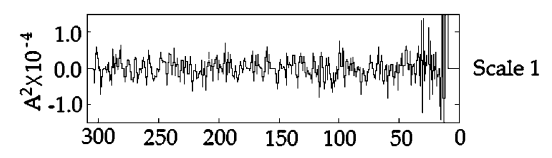

(b)

Fig. 7. (a) The result of the multi-resolution decomposition for the whole plant of Macromibiuma ferriei Card. Sz Ther. FT-IR spectra with DWT. (b) The result of the multi-resolution decomposition for the whole plant of Macromitrium syntrichophyllum Ther. Etp. Vard. FT-IR spectra with DWT. 


\subsection{The classification of species in Sect. Ptychomitrium Fueror.}

The same techniques, namely FSD and DWT, are also applied to the classification of Ptychomitrium sinense (Mitt.) Jaeg., Ptychomitrium dentatum (Mitt.) Jaeg. and Ptychomitrium polyphylloides (C. Muell.) Par. The spectra after the treatment of FSD are shown in Fig. 8. As shown in Fig. 8, all the FT-IR spectra are transformed by FSD to enhance the differences between the whole plants of Ptychomitrium sinense (Mitt.) Jaeg., Ptychomitrium dentatum (Mitt.) Jaeg. and Ptychomitrium polyphylloides (C. Muell.) Par. It is noted that the FSD spectra of Ptychomitrium sinense (Mitt.) Jaeg. and Ptychomitrium dentatum (Mitt.) Jaeg. have close absorbance and it is difficult to distinguish them. Compared with the FSD spectra, the amide I exists in Ptychomitrium sinense (Mitt.) Jaeg. and Ptychomitrium dentatum (Mitt.) Jaeg., but not in Ptychomitrium polyphylloides (C. Muell.) Par., or in Macromibiuma ferriei Card. Sz Ther., and it is correspondingly easy to make a distinction between Ptychomitrium polyphylloides (C. Muell.) Par. and the others. The difference between Ptychomitrium sinense (Mitt.)

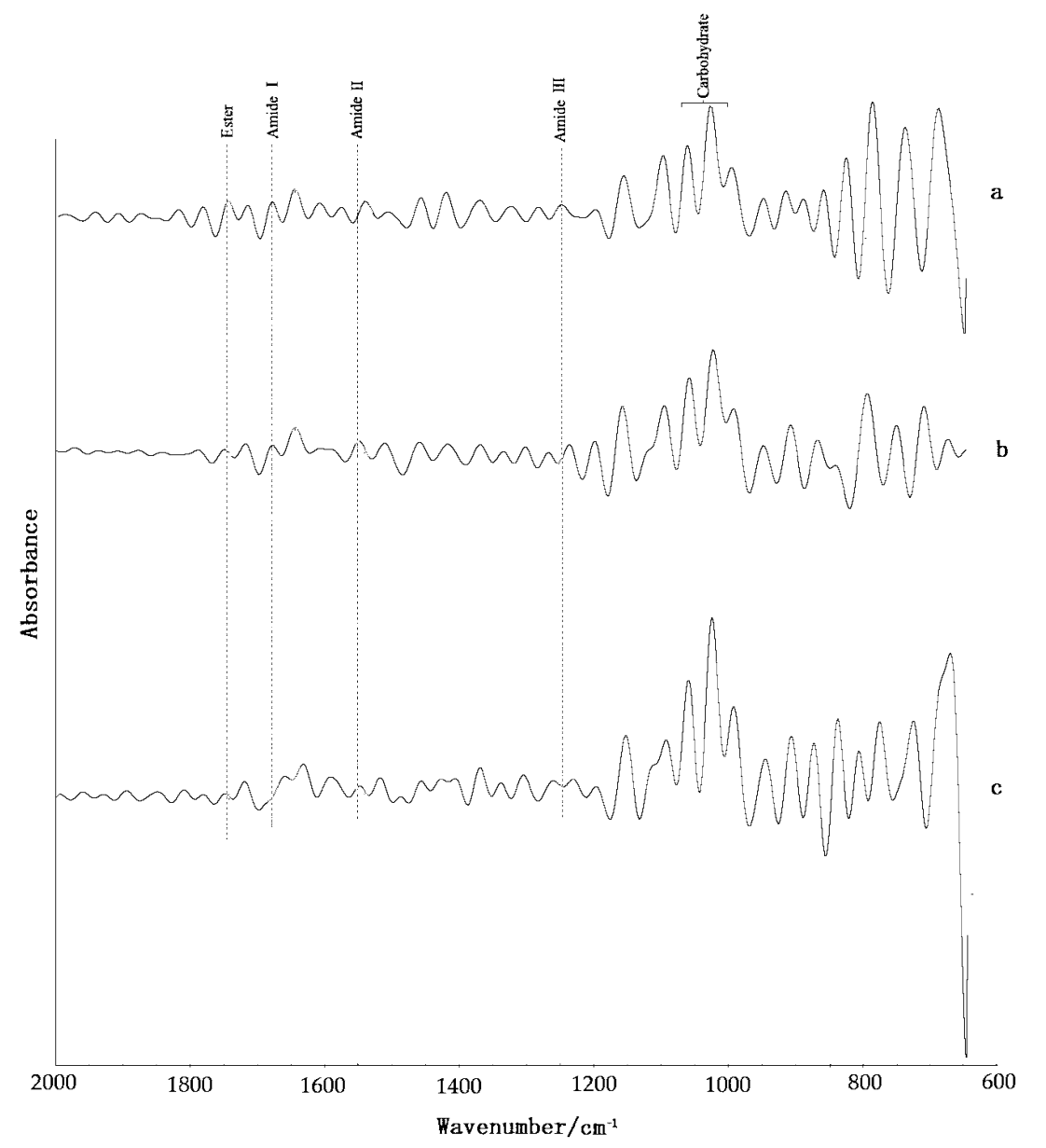

Fig. 8. Fourier self-deconvolution FT-IR spectra in the $2000-650 \mathrm{~cm}^{-1}$ region in the whole plants of three species in Sect. Ptychomitrium Fueror. Characteristic group frequencies are indicated at the top. (a) Ptychomitrium sinense (Mitt.) Jaeg. (b) Ptychomitrium dentatum (Mitt.) Jaeg. (c) Ptychomitrium polyphylloides (C. Muell.) Par. 

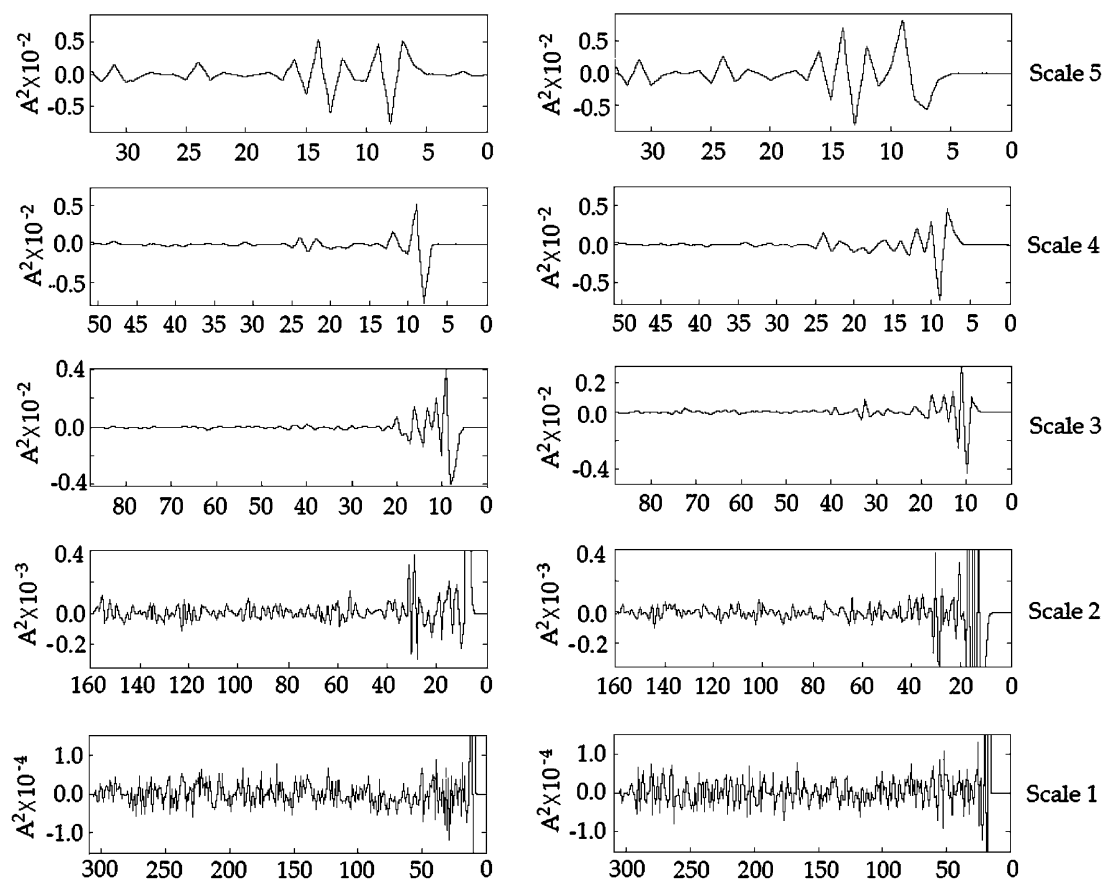

(a)

(b)

Fig. 9. (a) The result of the multi-resolution decomposition for the whole plant of Ptychomitrium sinense (Mitt.) Jaeg. FT-IR spectra with DWT. (b) The result of the multi-resolution decomposition for the whole plant of Ptychomitrium dentatum (Mitt.) Jaeg. FT-IR spectra with DWT.

Jaeg.and Ptychomitrium dentatum (Mitt.) Jaeg. is relatively little. To distinguish the little difference in patterns caused by overlapping bands, based on the above analysis, we used DWT to extract the features of Ptychomitrium sinense (Mitt.) Jaeg. and Ptychomitrium dentatum (Mitt.) Jaeg. for further classification. The region used in feature extraction is the $2000-650 \mathrm{~cm}^{-1}$.

Figure 9 described the DWT coefficients of the whole plant of Ptychomitrium sinense (Mitt.) Jaeg. and Ptychomitrium dentatum (Mitt.) Jaeg., where scales 1-5 respectively demonstrates detailed information after decomposition. In Fig. 9, the DWT coefficients can effectively reflect the features of spectra. Among scale 1-5, scale 3-5 clearly showed an obvious difference between Ptychomitrium sinense (Mitt.) Jaeg. and Ptychomitrium dentatum (Mitt.) Jaeg. According to the great difference among the DWT coefficients of the whole plant of Ptychomitrium sinense (Mitt.) Jaeg. and Ptychomitrium dentatum (Mitt.) Jaeg., in scales 3-5, different species in the same genera and family can be identified simply, promptly and accurately. The results further verified that DWT can be applied to extract the features of the plants in the same family which have similar infrared absorptions and enhance the difference among them and allow plant classification.

\subsection{Comparison of different samples of Ptychomitrium sinense (Mitt.) Jaeg.}

Because the samples came from the same plant, their chemical components should be almost identical or not much difference at least. As shown in Fig. 10, the FT-IR spectra of three samples, which were col- 


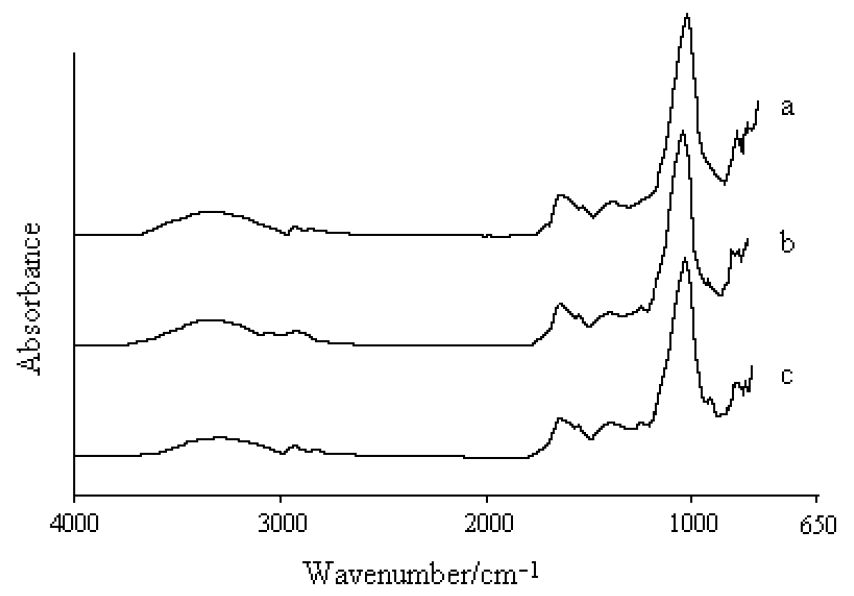

Fig. 10. The FT-IR spectra of three samples of Ptychomitrium sinense (Mitt.) Jaeg. (a) Beishan of Jinhua; (b) campus of Zhejiang Normal University; (c) Leshan of Leshan.

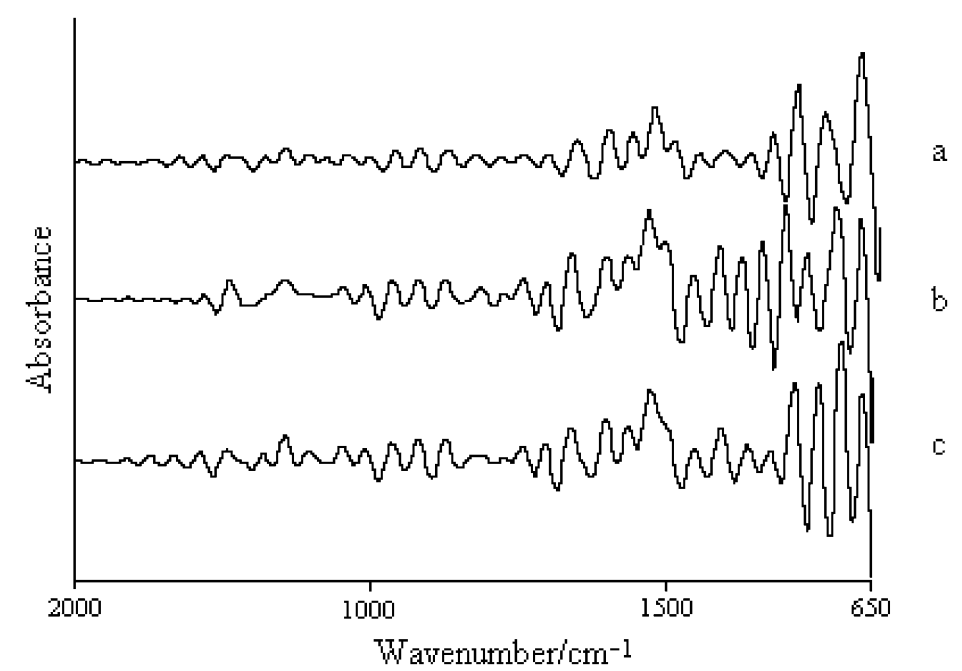

Fig. 11. The FSD spectra of three samples of Ptychomitrium sinense (Mitt.) Jaeg. (a) Beishan of Jinhua; (b) campus of Zhejiang Normal University; (c) Leshan of Leshan.

lected from Ptychomitrium sinense (Mitt.) Jaeg. from different areas, clearly indicated that intraspecific difference is almost unrecognizable by the naked eyes.

To verify the above results, the same techniques, namely FSD and DWT, were also applied to the classification of the different samples of Ptychomitrium sinense (Mitt.) Jaeg. As expected, the FSDprocessed spectra also indicated that they have very similar absorbance bands as shown in Fig. 11.

DWT was also used to extract the features of three samples for further classification.

Figure 12 described the DWT coefficients of three samples of Ptychomitrium sinense (Mitt.) Jaeg., scales 1-5 demonstrated respectively the detailed information after decomposition. Apparently, the DWT coefficients could effectively reflect the features of spectra. Among scales 1-5, scales 1-2 would 

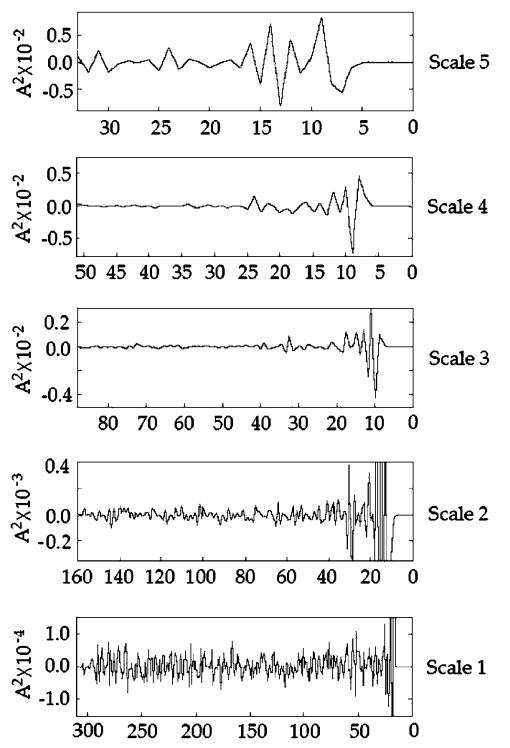

(a)
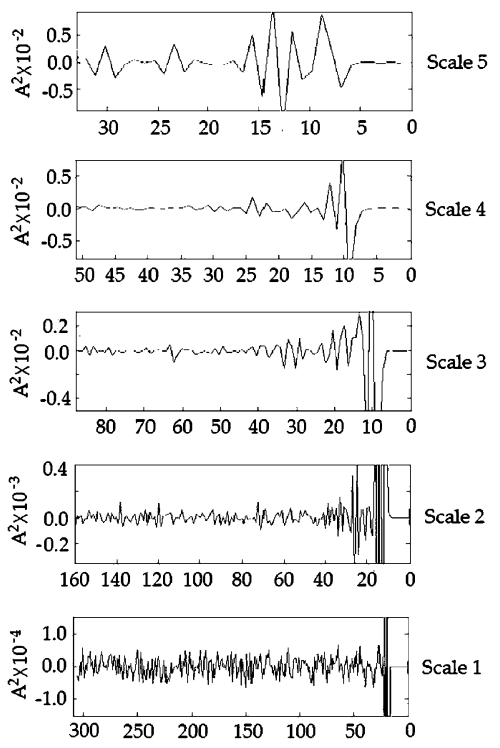

(b)
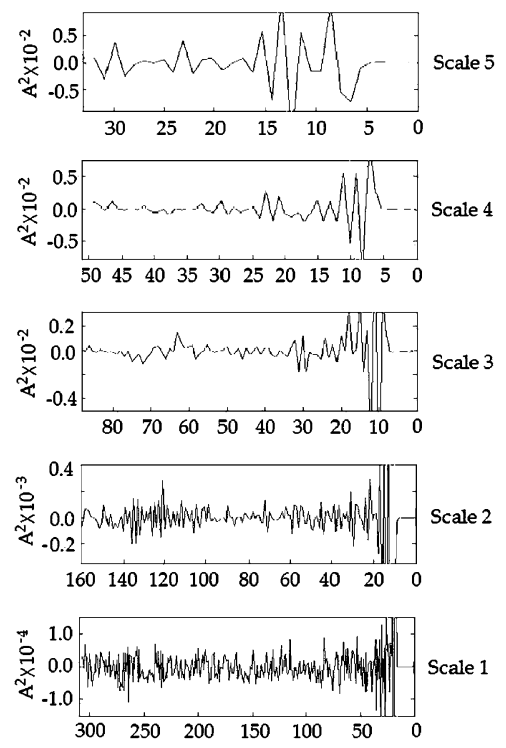

(c)

Fig. 12. The result of the multi-resolution decomposition for the three samples of Ptychomitrium sinense (Mitt.) Jaeg. FT-IR spectra with DWT. (a) Beishan of Jinhua; (b) campus of Zhejiang Normal University; (c) Leshan of Leshan.

not be used to analyze the intraspecific difference due to their relatively large noise, but scales 3-5 clearly showed very little intraspecific variation of Ptychomitrium sinense (Mitt.) Jaeg.

\section{Conclusion}

Due to the large variety, small habitus and few identifying characteristics of mosses, the classification of mosses is difficult in traditional plant taxonomy. In our study, we identify five kinds of moss species using chemical methods successfully based on their different chemical composition. FT-IR and HATR techniques are used to obtain the spectra of five mosses, which can give detailed information of different plants. CLA and PCA can be considered to identify the five moss species effectively. FSD and DWT are used to extract the features and enhance the differences between different species in the same family and genera whose FT-IR spectra are similar. The five moss species are successfully identified using HATR-FT-IR spectroscopy methods combined with FSD and DWT. By intraspecific comparison and interspecific comparison, the results from FSD and DWT further verified that interspecific variation was larger than intraspecific variation. The results show that HATR-FT-IR combined with chemometric methods for identification of mosses is efficient. The methods presented in this paper can result in significant advantages, particularly in terms of time that scientists, especially non-spectroscopists, have to spend on identification of mosses.

\section{Acknowledgements}

The authors would like to thank Professor Shuiliang Guo and Mr. Jiawei Chen from Department of Biology, Shanghai Normal University, China for identifying of the plant samples. 


\section{References}

[1] K.L.A. Chan and S.G. Kazarian, Appl. Spectrosc. 62 (2008), 1095-1101.

[2] C.G. Cheng, J. Liu, W.Q. Cao, R.W. Zheng, H. Wang and C.J. Zhang, Vib. Spectrosc. 54 (2010), 50-55.

[3] C.G. Cheng, J. Liu, H. Wang and W. Xiong, Appl. Spectrosc. Rev. 45 (2010), 165-178.

[4] C.G. Cheng, J. Liu, C.J. Zhang, M.Z. Cai, H. Wang and W. Xiong, Appl. Spectrosc. Rev. 45 (2010), 148-164.

[5] C.G. Cheng, Y.M. Ruan and B.L. Li, Spectrosc. Spect. Anal. 24 (2004), 1355-1358.

[6] C.G. Cheng, Y.M. Tian and W.Y. Jin, Spectroscopy - Int. J. 22 (2008), 397-404.

[7] C.G. Cheng, X.H. Wu and S.Q. Wang, Chin. J. Anal. Chem. 32 (2004), 1529-1531.

[8] C.G. Cheng, W. Xiong and W.Y. Jin, Chin. J. Anal. Chem. 37 (2009), 676-680.

[9] Q.H. Hong, C.G. Cheng, Z.F. Cheng and D.T. Li, Spectrosc. Spect. Anal. 27 (2007), 283-286.

[10] H.F. Lv, J.B. Shen, X.Y. Lin and J.L. Fu, Taxon 57 (2008), 1274-1288.

[11] Z. Movasaghi, S. Rehman and I. ur Rehman, Appl. Spectrosc. Rev. 43 (2008), 134-179.

[12] S. Prabhakar, A.R. Mohanty and A.S. Sekhar, Tribol. Int. 35 (2002), 793-800.

[13] The Pharmacopoeia Committee of the People's Republic of China, Chinese Pharmacopoeia, Vol. 1, Chemical Industrial Publishing House, Beijing, 2010.

[14] Y. Tuo, P. Huang, Y. Ke, S.L. Fan, Q.Y. Lu, B. Xin and Z.Y. Wang, Appl. Spectrosc. 64 (2010), 268-274.

[15] C.J. Zhang and C.G. Cheng, Spectroscopy - Int. J. 22 (2008), 371-386. 


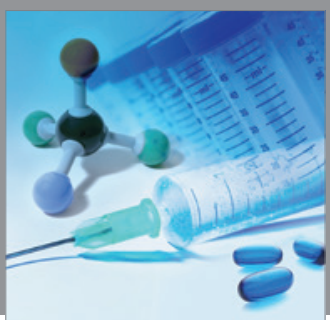

International Journal of

Medicinal Chemistry

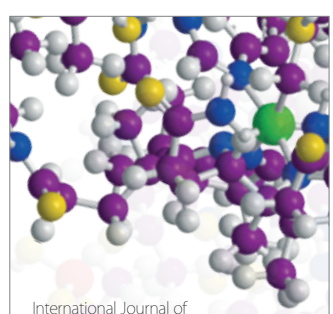

Carbohydrate Chemistry

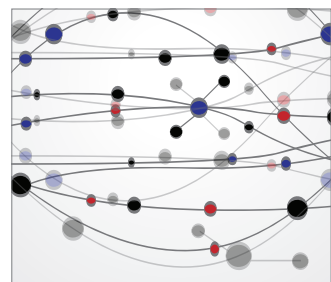

The Scientific World Journal
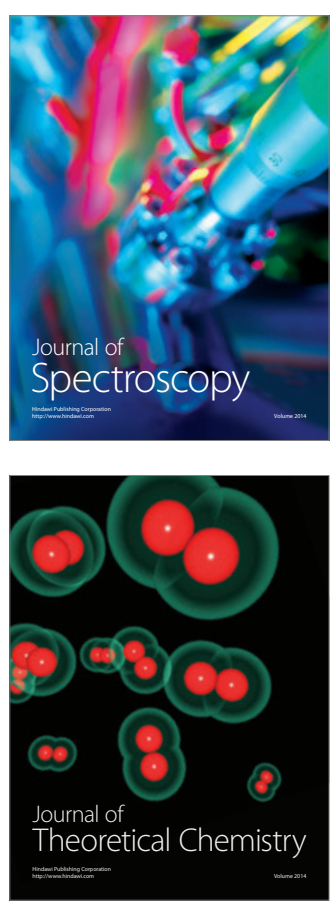
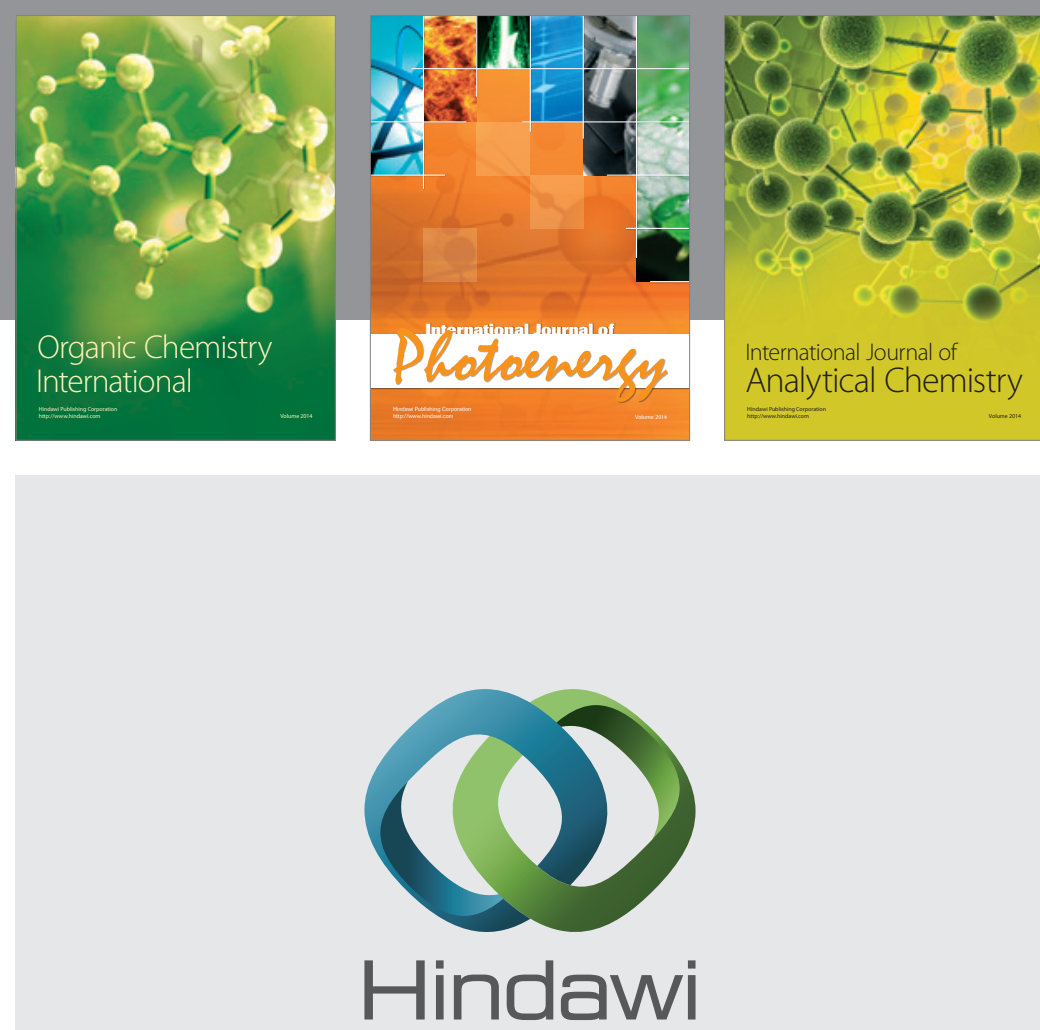

Submit your manuscripts at

http://www.hindawi.com
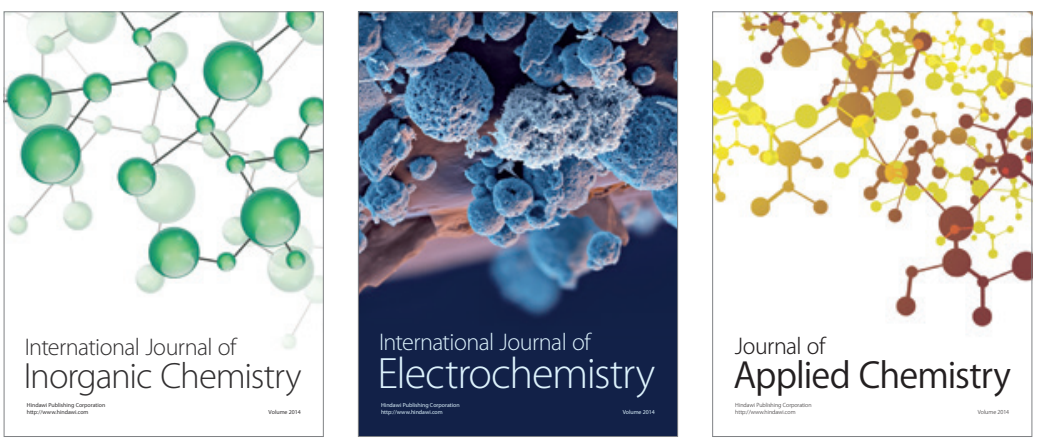

Journal of

Applied Chemistry
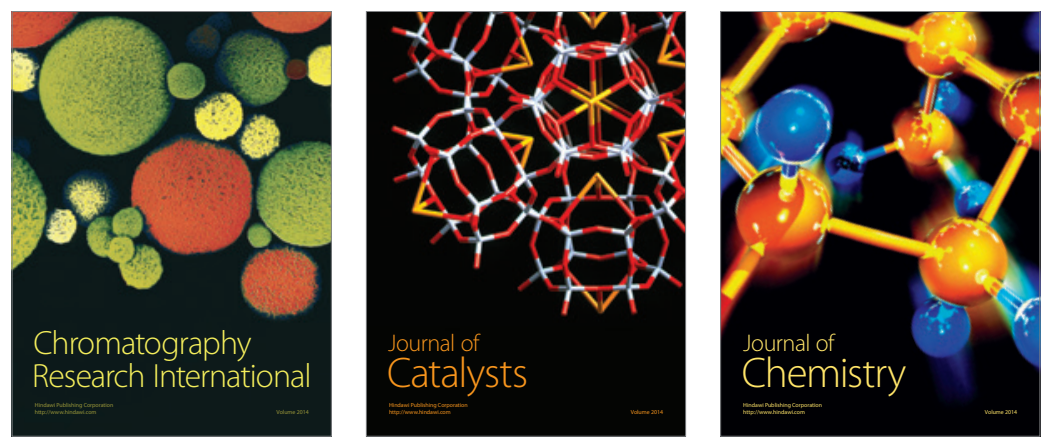
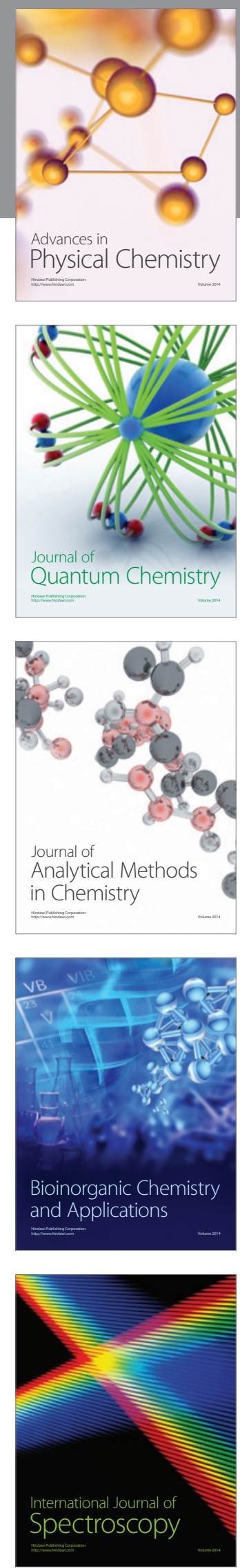